\title{
SIRT1 suppresses PMA and ionomycin-induced ICAM-1 expression in endothelial cells
}

\author{
JIA YuYan, GAO Peng, CHEN HouZao*, WAN YanZhen, ZHANG Ran, ZHANG ZhuQin, \\ YANG RuiFeng, WANG Xu, XU Jing \& LIU DePei*
}

State Key Laboratory of Medical Molecular Biology, Department of Biochemistry and Molecular Biology, Institute of Basic Medical Sciences, Chinese Academy of Medical Sciences \& Peking Union Medical College, Beijing, 100005, People's Republic of China

\begin{abstract}
Intercellular adhesion molecule-1 (ICAM-1) plays an important role in the recruitment of leukocytes to the endothelium, which causes inflammation and initiation of atherosclerosis. We have previously shown that endothelium-specific over-expression of class III deacetylase SIRT1 decreases atherosclerosis. We therefore addressed the hypothesis that SIRT1 suppresses ICAM-1 expression in the endothelial cells. Here, we found that expression of SIRT1 and ICAM-1 was significantly induced by PMA and ionomycin (PMA/Io) in human umbilical vein endothelial cells (HUVECs). Adenovirus-mediated over-expression of SIRT1 significantly inhibited PMA/Io-induced ICAM-1 expression in HUVECs. Knockdown of SIRT1 by RNA interference (RNAi) resulted in increased expression of ICAM-1 in HUVECs. Luciferase report assay showed that over-expression of SIRT1 suppressed ICAM-1 promoter activity both in basic and in PMA/Io-induced conditions. We further found that SIRT1 was involved in transcription complex binding on the ICAM-1 promoter by chromatin immunoprecipitation (ChIP) assays. Furthermore, SIRT1 RNAi increased NF-kB p65 binding ability to the ICAM-1 promoter by ChIP assays. Overall, these data suggests that SIRT1 inhibits ICAM-1 expression in endothelial cells, which may contribute to its anti-atherosclerosis effect.
\end{abstract}

\section{SIRT1, ICAM-1, PMA and ionomycin}

Citation: Jia Y Y, Gao P, Chen H Z, et al. SIRT1 suppresses PMA and ionomycin-induced ICAM-1 expression in endothelial cells . Sci China Life Sci, 2013, 56: 19-25, doi: 10.1007/s11427-012-4407-7

Leukocyte recruitment and expression of pro-inflammatory cytokines characterize early atherogenesis and malfunction of inflammatory mediators mutes atheroma formation in mice [1]. As the first barrier of blood vessels, the endothelium plays an extremely important role in maintaining vessel homeostasis [2]. Various leukocyte adhesion molecules increase accompanied by inflammatory activation in endothelial cells, which contribute to leukocyte recruitment in atherosclerosis-susceptible mice [3]. Thus, inhibition of leukocyte recruitment by decreasing the expression of adhesion molecules is important for preventing initiation of atherosclerosis.

*Corresponding author (email: houzao@gmail.com; liudp@pumc.edu.cn)
The expression of adhesion molecules such as intercellular adhesion molecule-1 (ICAM-1) is increased in atherosclerotic lesions [4]. Gene deletion of ICAM-1 resulted in significant reduction in monocyte recruitment to atherosclerotic lesions in ApoE-deficient mice [3]. ICAM-1 is upregulated in response to inflammatory stimuli such as interleukin-1 beta (IL-1 $\beta$ ), tumor necrosis factor-alpha (TNF- $\alpha$ ), LPS and PMA [5]. The divalent cation calcium ionophore ionomycin (Io) interacts synergistically with PMA but not with cytokines or LPS in upregulating ICAM-1 [5]. The combination of PMA and Io (PMA/Io) is routinely used as a T-cell receptor (TCR)-independent model to study T-cell activation and proliferation. PMA/Io mimics the phospholipase $\mathrm{C}$-driven activation of protein 
kinase $\mathrm{C}$ and cytosolic $\mathrm{Ca}^{2+}$ increase and induces activation of NF- $\kappa \mathrm{B}, \mathrm{NFAT}$ and AP-1. NF- $\kappa \mathrm{B}$ is of special interest in endothelial cells since it drives the expression of important adhesion molecules, such as ICAM-1, which recruit blood monocytes to atherosclerotic lesions [6]. NFAT cooperated with NF- $\kappa$ B to regulate thrombin-induced ICAM-1 gene expression by binding to the intronic $\mathrm{NF}-\kappa \mathrm{B}$ site in the ICAM-1 gene [7].

SIRT1, a well-known mammalian sirtuin, is involved in aging and age-related diseases [8]. SIRT1 has been described as a "guardian at the gates of adipose tissue inflammation", playing an important role in anti-inflammatory processes [9]. SIRT1 inhibits inflammatory pathways in macrophages and modulates insulin sensitivity [10]. SIRT1 acts to protect the heart from hypertrophy, metabolic dysregulation and inflammation [11]. Moreover, SIRT1 inhibits $\mathrm{NF}-\kappa \mathrm{B}$ and $\mathrm{AP}-1$ transcriptional activity and the expression of downstream inflammatory genes [12-15]. We have previously shown that transgenic mice that overexpress SIRT1 in the vascular endothelium have better endothelium-dependent vasodilation and fewer atherosclerotic lesions when fed a high-fat diet [16]. Recently, expression of ICAM-1 was increased in atherosclerotic plaques of Ap$\mathrm{oE}^{-/-} \mathrm{SIRT}^{+/-}$compared with $\mathrm{ApoE}^{-/-} \mathrm{SIRT1}^{+/+}$mice [17]. However, the mechanism by which SIRT1 regulates ICAM-1 expression remains unknown.

In the present study, we found that over-expression of SIRT1 significantly decreased PMA/Io-induced ICAM-1 expression in human umbilical vein endothelial cells (HUVECs). Knockdown of SIRT1 by RNA interference (RNAi) remarkably up-regulated ICAM-1 expression in HUVECs. Moreover, we found that SIRT1 significantly inhibited both basic and PMA/Io-induced ICAM-1 promoter activity. SIRT1 was involved in transcription complex binding on the ICAM-1 promoter by chromatin immunoprecipitation (ChIP) assays. Furthermore, SIRT1 RNAi increased NF- $\kappa \mathrm{B}$ p65 binding ability to the ICAM-1 promoter by ChIP assays. These data suggests that SIRT1 inhibits ICAM-1 expression in endothelial cells, which may contribute to its anti-atherosclerosis effect.

\section{Materials and methods}

\subsection{Drugs}

PMA (Cat \#P1585), Ionomycin (Cat \#I0634), Cyclosporin A (Cat \#C1832), resveratrol (Cat \#R5010) and Sirtinol (Cat \#S7942) were purchased from Sigma-Aldrich.

\subsection{Plasmids, HUVECs culture and adenovirus gener- ation}

SIRT1 expression vector is a gift from Dr. Ishikawa [18].
Endothelial cells were freshly isolated from human umbilical cord veins as described previously [16] and cultured in M200 medium (Cascade Biologics Inc., Portland, Oregon, USA). The growth medium was changed every two days until $>80 \%$ cells reached confluence. Cells between the $3 \mathrm{rd}$ and the 6th passages were grown in mono-layers in a humidified atmosphere of $5 \% \mathrm{CO}_{2}$ at $37^{\circ} \mathrm{C}$, and used for experiments at $>80 \%$ confluence. Replication-defective adenoviral vectors expressing SIRT1 (Ad-SIRT1) or a green fluorescent protein control (Ad-GFP) were prepared using the AdEasy vector kit (Quantum Biotechnologies) as described in the supplier's protocol. The adenovirusmediated knockdown of SIRT1 (Ad-SIRT1 RNAi) and a control vector (Ad-U6) were generated using the same system. HUVECs were infected with the above adenovirus for $24 \mathrm{~h}$ and were cultured in fresh M200 medium for further treatment.

\subsection{Reverse transcription and real-time PCR}

Total RNA was extracted from cells using Trizol (Invitrogen) according to the manufacturer's instructions. Two micrograms of total RNA were used to synthesize first-strand cDNA with M-MuLV reverse transcriptase (New England BioLabs) using random primers. Real-time PCR was performed using the BioRad iCycler iQ5 Real-Time PCR Detection System with the Quantitect SYBR Green One-Step RT-PCR Kit (QIAGEN). Fluorescence curves were analyzed with iCycler iQ5 Optical System Software (version 2.0). Primer sequences for specific genes are presented in Table S1.

\subsection{Western blotting analysis}

HUVEC protein was extracted with Radioimmunoprecipitation Assay (RIPA) buffer $\left(25 \mathrm{mmol} \mathrm{L}{ }^{-1}\right.$ Tris- $\mathrm{HCl} \mathrm{pH} \mathrm{7.6,}$ $150 \mathrm{mmol} \mathrm{L}^{-1} \mathrm{NaCl}, 1 \% \mathrm{NP}-40,1 \%$ sodium deoxycholate and $0.1 \% \mathrm{SDS}$ ). After lysis on an ice for $0.5 \mathrm{~h}$, samples were sonicated and centrifuged at $4{ }^{\circ} \mathrm{C}$ at $12000 \mathrm{r} \mathrm{min}^{-1}$ for $0.5 \mathrm{~h}$. The supernatants were transferred into fresh tubes and protein concentrations were determined by the bicinchoninic acid (BCA) method. Equal amounts of protein (20 $\mu \mathrm{g} /$ lane) were separated by SDS-PAGE and transferred onto polyvinylidene difluoride membranes (Millipore). After being blocked, the filters were incubated with the following primary antibodies: anti-SIRT1 (Santa Cruz Biotechnology, Cat \#sc-15404), anti-ICAM-1 (Cell Signal Technology, Cat \#4915). After being washed and incubated with the appropriate horseradish peroxidase-conjugated secondary antibody (Santa Cruz Biotechnology), the immune complexes were visualized with a chemiluminescence reagent. Western blotting was quantified densitometrically with Quantity One software (Bio-Rad), and the intensity values were normalized to anti-GAPDH. 


\subsection{Transfection and luciferase assays}

HEK293 cells were grown as recommended by ATCC and were transfected with Lipofectamine 2000 (Invitrogen) according to the manufacturer's instructions. Luciferase assays were performed using a dual luciferase reporter assay system (Promega). Luciferase activity was normalized by transfection efficiency using pRL-CMV reporter (Promega) as an internal control. The results are expressed as percentages of relative luciferase activity of the control group.

\subsection{ChIP}

Chromatin immunoprecipitation (ChIP) assays were performed in HUVECs as described [19]. A rabbit polyclonal antibody for SIRT1 (Santa Cruz Biotechnology, Cat \#sc-15404) was used in ChIP assay. Control immunoprecipitations were carried out with nonimmune normal rabbit IgG (Santa Cruz Biotechnology, Cat \#sc-2027).

\subsection{Statistical analysis}

Data are expressed as mean \pm SD. Statistical analyses were performed using the two-tailed unpaired Student's $t$-test to determine statistical significances between the groups. A $P$-value less than 0.05 was considered significant.

\section{Results}

\subsection{SIRT1 and ICAM-1 expression is induced by PMA/Io in HUVECs}

Considering that the ICAM-1 promoter has binding sites for NF- $\kappa$ B, NFAT and AP-1, we treated HUVECs with PMA/Io, which is known to induce activation of transcription factors NF- $\kappa$ B, NFAT and AP-1. Previous studies have shown that ionomycin (Io) interacted synergistically with PMA in upregulating ICAM-1 expression [5]. Here we repeated the results and found that ICAM-1 mRNA expression level was induced higher by PMA than by Io (Figure 1B). In contrast, SIRT1 mRNA level was significantly induced about to 3 -fold by Io, but was not significantly changed by PMA (Figure1A). The results showed that an Io-activated cacium-calcineurin-NFAT signal pathway played an important role in inducing SIRT1 expression. Io also interacted synergistically with PMA in inducing SIRT1 mRNA expression level about 3.6-fold (Figure 1A). Moreover, SIRT1 and ICAM-1 protein expression was also induced by PMA/Io in HUVECs (Figure 1C and D).

\subsection{Over-expression of SIRT1 suppresses ICAM-1 ex- pression in PMA/Io-treated HUVECs}

To study the effect of increased expression of SIRT1 on
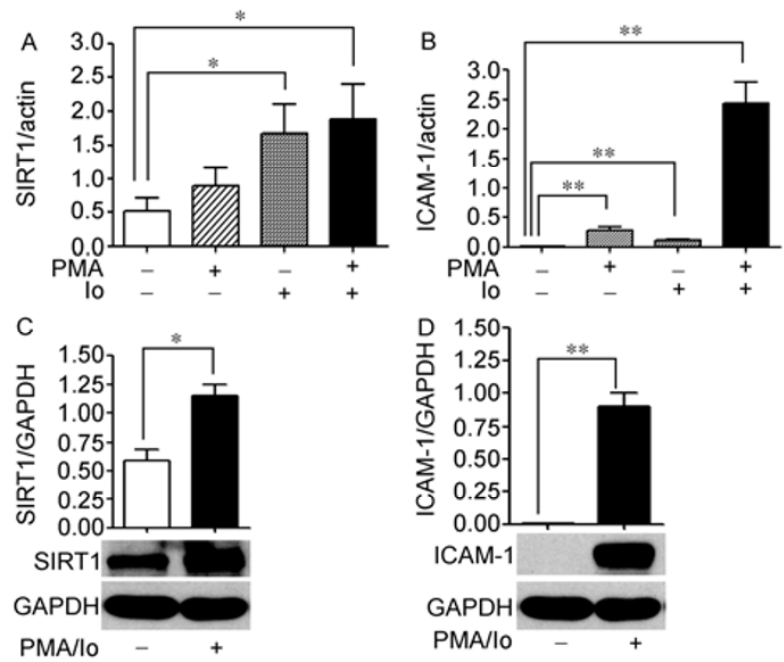

Figure 1 SIRT1 and ICAM-1 expression is significantly induced in HUVECs treated with PMA/Io. A and B, HUVECs were treated with PMA

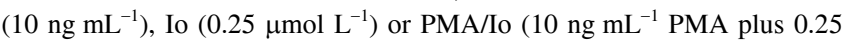

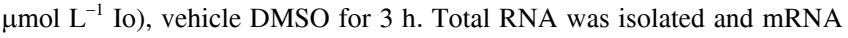
expression level for SIRT1 (A) and ICAM-1 (B) was analyzed by real-time $\mathrm{PCR}$ analysis. Data shown represent the mean $\pm \mathrm{SD}$ of triplicate samples of one representative of total three independent experiments. Relative mRNA unit is calculated by using actin as a reference gene. ${ }^{*}, P<0.05 ; * *, P<0.01$. $\mathrm{C}$ and D, HUVECs were treated with PMA/Io (10 $\mathrm{ng} \mathrm{mL}^{-1}$ PMA plus 0.25 $\mu \mathrm{mol} \mathrm{L}{ }^{-1}$ Io) for $3 \mathrm{~h}$. Protein expression was analyzed by Western blotting. Bar graphs show densitometric analysis of immunoblots of SIRT1 (C) and ICAM-1(D) protein. Data are presented as mean \pm SD of SIRT1/GAPDH and ICAM-1/ GAPDH expression ratio $(n=3)$. Immunoblots of SIRT1, ICAM-1 and GAPDH are representative of three independent experiments. $*, P<0.05$; **, $P<0.01$.

ICAM-1 expression, we over-expressed SIRT1 in PMA/Iotreated HUVECs. We found that over-expression of SIRT1 suppressed PMA/Io-induced expression of both ICAM-1 mRNA and protein in HUVECs to $60 \%$ and $50 \%$, respectively (Figure 2A). Similarly, SIRT1 activator resveratrol (RSV) suppressed ICAM-1 protein expression to $18 \%$ (Figure $2 \mathrm{~B})$.

To detect whether NFAT inhibition influences the suppressive effect of SIRT1 on ICAM-1 expression, we over-expressed SIRT1 in PMA/Io-treated HUVECs both in the presence and absence of NFAT inhibitor Cyclosporin A (CsA). We found that over-expression of SIRT1 suppressed ICAM-1 protein expression in HUVECs induced by PMA/Io either in presence or absence of CsA to $44 \%$ and $53 \%$, respectively (Figure 2C). The results showed that there was no significant effect of CsA on inhibition of ICAM-1 expression by SIRT1.

\subsection{Knockdown of SIRT1 upregulates ICAM-1 expres- sion in HUVECs}

To examine the effect of SIRT1 knockdown on ICAM-1 expression, we infected HUVECs with adenoviral vectors encoding SIRT1 RNAi (Ad-SIRT1 RNAi) or control (AdU6). Knockdown of SIRT1 by RNA interference (RNAi) 

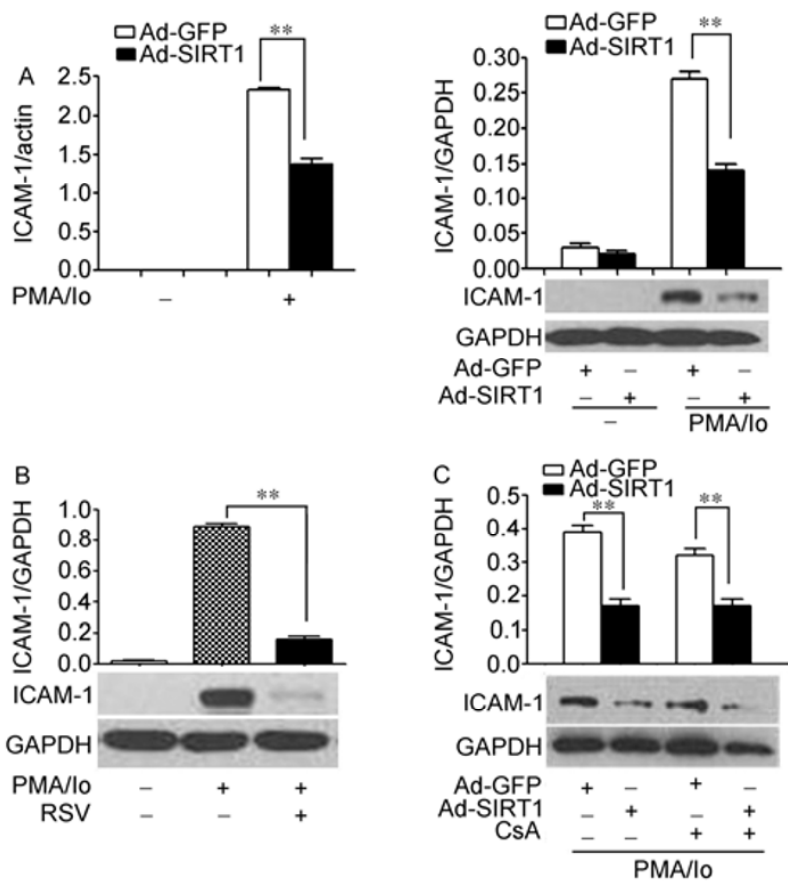

Figure 2 Over-expression of SIRT1 suppresses ICAM-1 expression in HUVECs treated with PMA/Io. A, HUVECs infected with adenoviral vectors encoding SIRT1 (Ad-SIRT1) or control Ad-GFP were treated with PMA/Io (10 ng mL ${ }^{-1}$ PMA plus $0.25 \mu \mathrm{mol} \mathrm{L}^{-1}$ Io) for $3 \mathrm{~h}$. Total RNA was isolated and mRNA level for ICAM-1 was analyzed by real-time PCR analysis (left). Relative mRNA unit is calculated by using actin as a reference gene. Protein expression was analyzed by Western blotting (right). Bar graphs show densitometric analysis of immunoblots of ICAM-1 protein. Data are presented as the mean \pm SD of ICAM-1/GAPDH expression ratio $(n=3)$. Immunoblots of ICAM-1 and GAPDH are representative of three independent experiments. $* *, P<0.01$. B, HUVECs were pretreated with resveratrol (RSV) $\left(30 \mu \mathrm{mol} \mathrm{L}^{-1}\right)$ or vehicle DMSO for $1 \mathrm{~h}$, then treated with PMA/Io (10 ng mL ${ }^{-1}$ PMA plus $0.25 \mu \mathrm{mol} \mathrm{L} \mathrm{L}^{-1}$ Io) for another $3 \mathrm{~h}$. Protein expression was analyzed by Western blotting. Bar graphs show densitometric analysis of immunoblots of ICAM-1 protein. Data are presented as the mean \pm SD of ICAM-1/GAPDH expression ratio $(n=3)$. Immunoblots of ICAM-1 and GAPDH are representative of three independent experiments. **, $P<0.01$. C, HUVECs infected with adenoviral vectors encoding SIRT1 (Ad-SIRT1) or control Ad-GFP for $24 \mathrm{~h}$, then pretreated with CsA $\left(1 \mu \mathrm{mol} \mathrm{L}{ }^{-1}\right)$ or vehicle DMSO for $1 \mathrm{~h}$ and treated with PMA/Io (10 ng mL $\mathrm{m}^{-1}$ PMA plus $0.25 \mu \mathrm{mol} \mathrm{L}{ }^{-1}$ Io) for another $3 \mathrm{~h}$. Protein expression was analyzed by Western blotting. Bar graphs show densitometric analysis of immunoblots of ICAM-1 protein. Data are presented as the mean \pm SD of ICAM-1/GAPDH expression ratio $(n=3)$. Immunoblots of ICAM-1 and GAPDH are representative of three independent experiments. **, $P<0.01$.

upregulated ICAM-1 expression at both the mRNA and protein levels in HUVECs to 2-fold and 3-fold, respectively (Figure 3A). Similarly, SIRT1 inhibitor Sirtinol significantly upregulated ICAM-1 expression in HUVECs to 37.5-fold (Figure 3B).

\subsection{SIRT1 inhibits ICAM-1 promoter activity induced by PMA/Io}

To further examine whether SIRT1 inhibits ICAM-1 promoter activity, we performed assays using luciferase report- er containing the ICAM-1 promoter (ICAM-1-Luc). Luciferase report assay showed that over-expression of SIRT1 suppressed ICAM-1 promoter activity both in basic and PMA/Io-induced conditions (Figure 4).

\subsection{SIRT1 is involved in transcription complex binding on the ICAM-1 promoter in HUVECs}

The first $1.3 \mathrm{~kb}$ upstream of the ICAM-1 transcription start site are required for ICAM-1basal expression and regulation by inflammatory stimuli [20]. To explore whether SIRT1 is involved in transcription complex binding on the ICAM-1 promoter, we performed ChIP assays in HUVECs on a 1.3 $\mathrm{kb}$ region of the ICAM-1 promoter upstream of the transcription start site, using semiquantitative PCR (qPCR) with primers for regions $(\mathrm{R})$ named 1 (-1297 to $-1020 \mathrm{bp}) ; 2$ ( -1033 to $-793 \mathrm{bp}) ; 3$ ( -814 to $-551 \mathrm{bp}) ; 4$ ( -577 to -271 bp); and 5 ( -287 to $+16 \mathrm{bp})$. This showed that an increase in the amount of SIRT1 bound to R5, compared with the others regions (Figure 5A). These data indicated that SIRT1 was involved in transcription complex binding on the ICAM-1 promoter in a region detected by the $\mathrm{R} 5$ primers $(-287$ to $+16 \mathrm{bp})$.

$\mathrm{NF}-\kappa \mathrm{B}$ is deacetylated and transcriptionally suppressed by SIRT1 and binds at site -187 to -178 bp of the ICAM- 1 promoter [12,21]. To examine whether NF- $\kappa \mathrm{B}$ is involved in the effect of SIRT1 on regulating expression of ICAM-1, we detected NF- $\kappa \mathrm{B}$ p65 binding ability on ICAM-1 promoter region 5 ( -287 to +16 bp) by ChIP analysis. As shown in Figure 5, SIRT1 RNAi increases NF- $\kappa$ B p65 binding ability on ICAM-1 promoter region 5 (Figure 5B).

\section{Discussion}

Here, we found that expression of SIRT1 and ICAM-1 was significantly increased by PMA/Io in HUVECs. Overexpression of SIRT1 significantly inhibited PMA/Io- induced ICAM-1 expression in HUVECs. Moreover, SIRT1 suppressed ICAM-1 promoter activity both in basic and PMA/Io stimulated conditions. We also found that SIRT1 was involved in transcription complex binding on the ICAM-1 promoter by ChIP assays. Furthermore, SIRT1 RNAi increased NF- $\kappa$ B p65 binding ability to the ICAM-1 promoter by ChIP assays.

SIRT1 has been pointed as a key regulator of vascular endothelial homeostasis controlling angiogenesis, vascular tone and endothelial dysfunction [22]. More evidence has pointed out that SIRT1 is a key inducible factor in response to inflammatory stimulation. For example, we have previously shown that oxLDL and $\mathrm{H}_{2} \mathrm{O}_{2}$ treatment increased SIRT1 protein levels in HUVECs $[16,18]$, and TNF- $\alpha-$ induced SIRT1 expression in vascular smooth muscle cells 

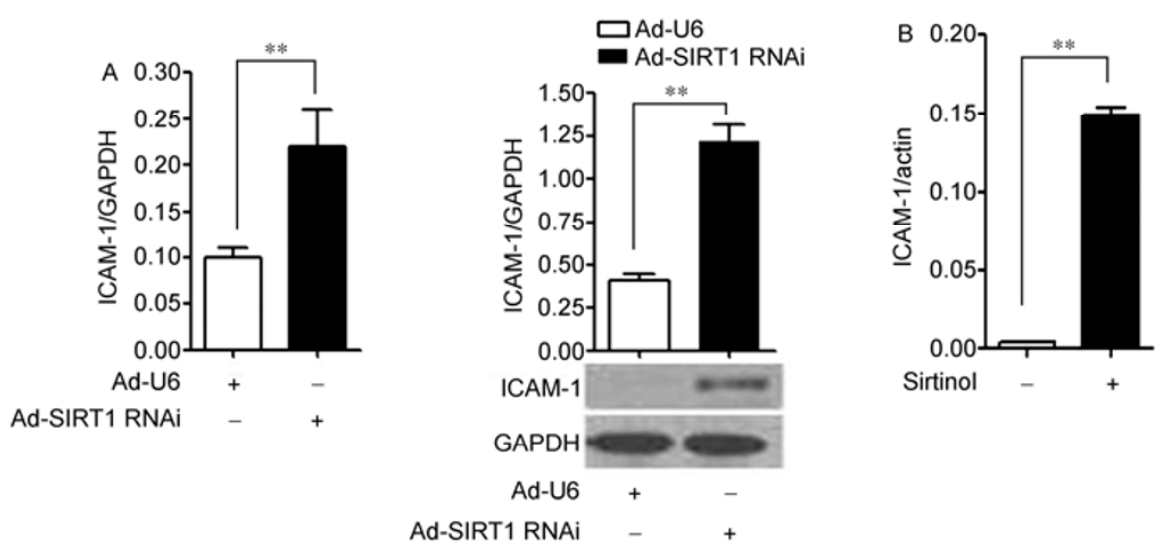

Figure 3 Knockdown of SIRT1 upregulates ICAM-1 expression in HUVECs. A, HUVECs were infected with adenoviral vectors SIRT1 RNAi (Ad-SIRT1 RNAi) or control Ad-U6. Total RNA was isolated and mRNA level for ICAM-1 was analyzed by real-time PCR analysis (left). Data shown represent the mean $\pm \mathrm{SD}$ of triplicate samples of one representative of total three independent experiments. Relative mRNA unit is calculated by using GAPDH as a reference gene. Protein expression was analyzed by Western blotting (right). Bar graphs show densitometric analysis of immunoblots of ICAM-1 protein. Data are presented as the mean \pm SD of ICAM-1/GAPDH expression ratio $(n=3)$. Immunoblots of ICAM-1 and GAPDH are representative of three independent experiments. **, $P<0.01$. B, HUVECs were treated with Sirtinol $\left(25 \mu \mathrm{mol} \mathrm{L}^{-1}\right)$ or vehicle DMSO for $1 \mathrm{~h}$. Total RNA was isolated and mRNA level for ICAM-1 was analyzed by real-time PCR analysis. Data shown represent the mean \pm SD of triplicate samples of one representative of total three independent experiments. Relative mRNA unit is calculated by using actin as a reference gene. ${ }^{* *}, P<0.01$.

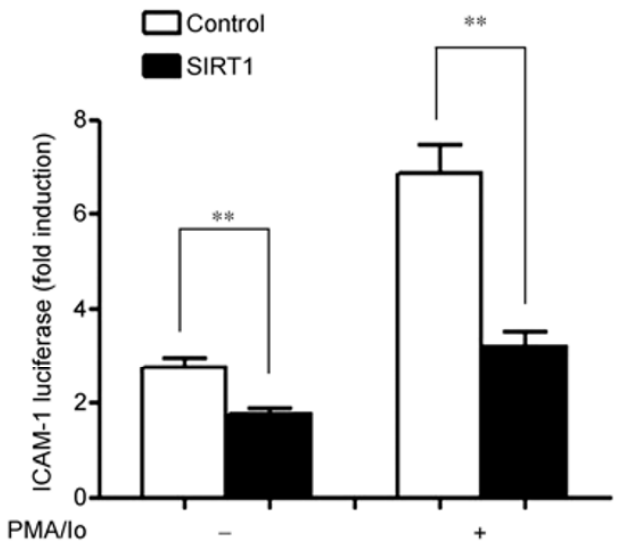

Figure 4 Over-expression of SIRT1 suppresses ICAM-1 promoter activity. HEK293 cells were transiently transfected with $0.1 \mu \mathrm{g}$ ICAM-1 luciferase reporter (ICAM-1-Luc), $30 \mathrm{ng}$ pRL-CMV, and $0.3 \mu \mathrm{g}$ SIRT1 expression vectors or control (pcDNA3.1) for $24 \mathrm{~h}$, then treated with PMA/Io (10 $\mathrm{ng} \mathrm{mL} \mathrm{m}^{-1}$ PMA plus $0.25 \mu \mathrm{mol} \mathrm{L} \mathrm{L}^{-1}$ Io) or vehicle DMSO for $3 \mathrm{~h}$. The relative luciferase activities are presented as mean $\pm \mathrm{SD}$ of triplicate samples and are representative of three independent experiments. **, $P<0.01$.

(VSMCs) [23]. Here, we found that the SIRT1 level was induced by PMA/Io in HUVECs. It suggests that SIRT1 has a compensatory upregulation in endothelial cells in response to inflammatory factors. Moreover, expression of ICAM-1 was found to be increased in atherosclerotic plaques of $\mathrm{ApoE}^{-/-} \mathrm{SIRT}^{+/-}$mice compared with $\mathrm{ApoE}^{-/-} \mathrm{SIRT1}^{+/+}$ mice [17]. Furthermore, we demonstrated that SIRT1 significantly inhibited PMA/Io-induced ICAM-1 expression in HUVECs to $50 \%$. Overall, these data suggests that SIRT1 inhibits ICAM-1 expression in the endothelial cells, which may contribute to its anti-atherosclerosis effect.

The combination of PMA and Io activated these signals that mimic the phospholipase $\mathrm{C}$-driven activation of protein kinase $\mathrm{C}$ and increase in cytosolic $\mathrm{Ca}^{2+}$ and induce the transcription factors NF- $\mathrm{KB}$, NFAT and AP-1. Our previous work demonstrated SIRT1 suppresses the transcriptional activity of AP-1 [15]. Moreover, SIRT1 inhibits NF- $\mathrm{kB}$ transcriptional activity [12]. NF- $\mathrm{\kappa B}$ plays essential roles in transcriptional regulation of ICAM-1 in endothelial cells and binds at site -187 to -178 bp of the ICAM- 1 promoter [21]. In addition, ERK, JNK, AP-1 and NF- $\mathrm{kB}$ are all involved in interleukin-1-beta-induced ICAM-1 expression enhancing leukocyte adhesion in human rheumatoid arthritis synovial fibroblasts [24]. We found that SIRT1 bound the ICAM-1 promoter at the R5 region ( -287 to $+16 \mathrm{bp})$ and $\mathrm{NF}-\mathrm{KB}$ was included in the region. Moreover, SIRT1 inhibits PMA/Io induced NF- $\mathrm{KB}$ transcriptional activity and ICAM-1 promoter activity. SIRT1 is involved in transcription complex binding on the ICAM-1 promoter and SIRT1 RNAi increases NF- $\mathrm{kB}$ p65 binding ability to the ICAM-1 promoter. The data indicated that suppressive effect of SIRT1 on ICAM-1 expression was at least partly mediated

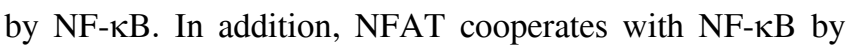
binding to the intronic NF-KB site on the ICAM-1 gene [7]. Our findings demonstrated that over-expression of SIRT1 inhibited ICAM-1 expression in PMA/Io-treated HUVECs both in the presence and absence of NFAT inhibitor CsA, although the extent of inhibition was not of statistical significance ( $44 \%$ and $53 \%$, respectively). This indicated that NFAT inhibition may not influence the effect of SIRT1 on inhibition of ICAM-1 expression. 

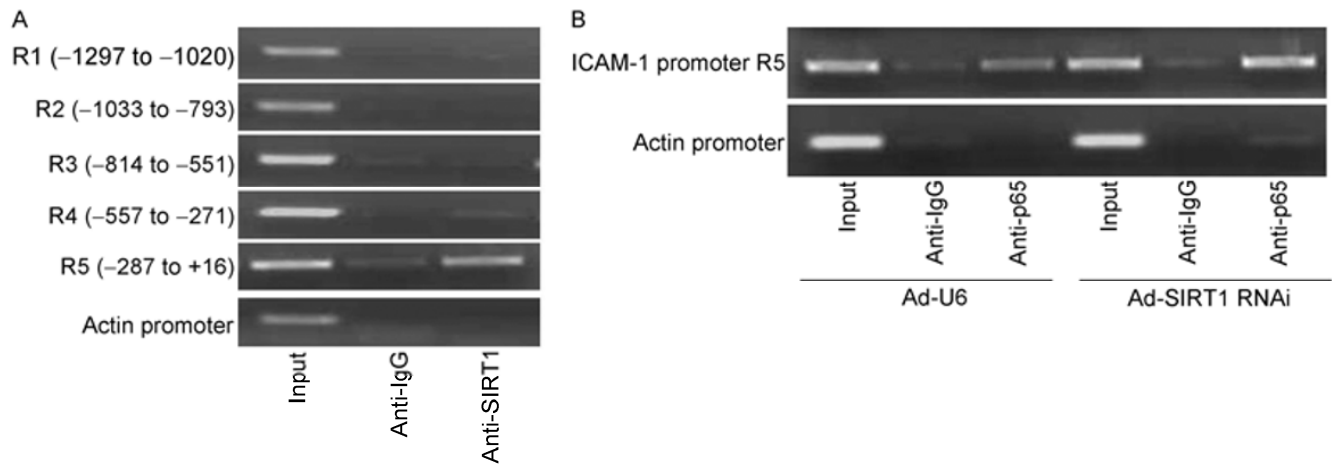

Figure 5 SIRT1 is involved in transcription complex binding on the ICAM-1 promoter. A, ChIP assays were performed with chromatin prepared from HUVECs. Chromatin was immunoprecipitated with normal rabbit IgG or antibody against SIRT1, and precipitated genomic DNA was an analyzed by semiquantitative PCR using primers for the specific ICAM-1 promoter region, respectively. B, HUVECs were infected with adenoviral vectors encoding SIRT1 RNAi (Ad-SIRT1 RNAi) or control Ad-U6 for 24 h. ChIP assays were performed with chromatin prepared from HUVECs. Chromatin was immunoprecipitated with normal rabbit IgG, antibody against p65, and precipitated genomic DNA was analyzed by semiquantitative PCR using primers for the specific ICAM-1 promoter region 5 ( -287 to $+16 \mathrm{bp})$.

In conclusion, our data provide evidence that SIRT1 significantly inhibited PMA/Io-induced ICAM-1 expression in endothelial cells. Therefore, inhibition of ICAM-1 expression by activation of SIRT1 may be a potential therapeutic strategy for inflammation in atherosclerosis.

This work was supported by National Natural Science Foundation of China (31271227, 31028005, 31021091) and National Basic Research Program of China (2011CB503902, 2012BAI39B03).

1 Libby P. Inflammation in atherosclerosis. Nature, 2002, 420: 868874

2 Libby P, Ridker P M, Hansson G K. Progress and challenges in translating the biology of atherosclerosis. Nature, 2011, 473: 317325

3 Collins R G, Velji R, Guevara N V, et al. P-selectin or intercellular adhesion molecule (ICAM)-1 deficiency substantially protects against atherosclerosis in apolipoprotein E-deficient mice. J Exp Med, 2000, 191: 189-194

4 Poston R N, Haskard D O, Coucher J R, et al. Expression of intercellular adhesion molecule-1 in atherosclerotic plaques. Am J Pathol, 1992, 140: 665-673

5 Myers C L, Desai S N, Schembri-King J, et al. Discriminatory effects of protein kinase inhibitors and calcium ionophore on endothelial ICAM-1 induction. Am J Physiol, 1992, 262: C365-373

$6 \mathrm{Kim}$ I, Moon S O, Kim S H, et al. Vascular endothelial growth factor expression of intercellular adhesion molecule 1 (ICAM-1), vascular cell adhesion molecule 1 (VCAM-1), and E-selectin through nuclear factor-kappa B activation in endothelial cells. J Biol Chem, 2001, 276: 7614-7620

7 Xue J, Thippegowda P B, Hu G, et al. NF-kappaB regulates thrombin-induced ICAM-1 gene expression in cooperation with NFAT by binding to the intronic NF-kappaB site in the ICAM-1 gene. Physiol Genomics, 2009, 38: 42-53

8 Blander G, Guarente L. The Sir2 family of protein deacetylases. Annu Rev Biochem, 2004, 73: 417-435

9 Cho K W, Lumeng C N. SirT1: a guardian at the gates of adipose tissue inflammation. Diabetes, 2011, 60: 3100-3102
10 Yoshizaki T, Schenk S, Imamura T, et al. SIRT1 inhibits inflammatory pathways in macrophages and modulates insulin sensitivity. Am J Physiol Endocrinol Metab, 2010, 298: E419-428

11 Planavila A, Iglesias R, Giralt M, et al. Sirt1 acts in association with PPARalpha to protect the heart from hypertrophy, metabolic dysregulation, and inflammation. Cardiovasc Res, 2011, 90: 276-284

12 Yeung F, Hoberg J E, Ramsey C S, et al. Modulation of NF-kappaBdependent transcription and cell survival by the SIRT1 deacetylase. EMBO J, 2004, 23: 2369-2380

13 Lagouge M, Argmann C, Gerhart-Hines Z, et al. Resveratrol improves mitochondrial function and protects against metabolic disease by activating SIRT1 and PGC-1alpha. Cell, 2006, 127: 1109-1122

14 Li X, Zhang S, Blander G, et al. SIRT1 deacetylates and positively regulates the nuclear receptor LXR. Mol Cell, 2007, 28: 91-106

15 Zhang R, Chen H Z, Liu J J, et al. SIRT1 suppresses activator protein- 1 transcriptional activity and cyclooxygenase- 2 expression in macrophages. J Biol Chem, 2010, 285: 7097-7110

16 Zhang Q J, Wang Z, Chen H Z, et al. Endothelium-specific overexpression of class III deacetylase SIRT1 decreases atherosclerosis in apolipoprotein E-deficient mice. Cardiovasc Res, 2008, 80: 191199

17 Stein S, Schafer N, Breitenstein A, et al. SIRT1 reduces endothelial activation without affecting vascular function in $\mathrm{ApoE}^{-/-}$mice. Aging (Albany NY), 2010, 2: 353-360

18 Takata T, Ishikawa F. Human Sir2-related protein SIRT1 associates with the bHLH repressors HES1 and HEY2 and is involved in HES1and HEY2-mediated transcriptional repression. Biochem Biophys Res Commun, 2003, 301: 250-257

19 Zhou S, Chen H Z, Wan Y Z, et al. Repression of P66Shc expression by SIRT1 contributes to the prevention of hyperglycemia-induced endothelial dysfunction. Circ Res, 2011, 109: 639-648

20 Voraberger G, Schafer R, Stratowa C. Cloning of the human gene for intercellular adhesion molecule 1 and analysis of its $5^{\prime}$-regulatory region. Induction by cytokines and phorbol ester. J Immunol, 1991, 147: 2777-2786

21 Ledebur H C, Parks T P. Transcriptional regulation of the intercellular adhesion molecule-1 gene by inflammatory cytokines in human endothelial cells. Essential roles of a variant NF-kappa B site and p65 homodimers. J Biol Chem, 1995, 270: 933-943

22 Potente M, Dimmeler S. Emerging roles of SIRT1 in vascular endothelial homeostasis. Cell Cycle, 2008, 7: 2117-2122 
23 Zhang $\mathrm{H} \mathrm{N}$, Li L, Gao P, Chen $\mathrm{H} \mathrm{Z}$, et al. Involvement of the p65/RelA subunit of NF-kappaB in TNF-alpha-induced SIRT1 expression in vascular smooth muscle cells. Biochem Biophys Res Commun, 2010, 397: 569-575
24 Yang C M, Luo S F, Hsieh H L, et al. Interleukin-1beta induces ICAM-1 expression enhancing leukocyte adhesion in human rheumatoid arthritis synovial fibroblasts: involvement of ERK, JNK, AP-1, and NF-kappaB. J Cell Physiol, 2010, 224: 516-526

Open Access This article is distributed under the terms of the Creative Commons Attribution License which permits any use, distribution, and reproduction in any medium, provided the original author(s) and source are credited.

\section{Supporting Information}

Figure S1 Over-expression of SIRT1 suppresses PMA/Io induced NF- $\kappa B$ transcriptional activity. HEK293 cells were transiently transfected with 0.1 $\mu$ g

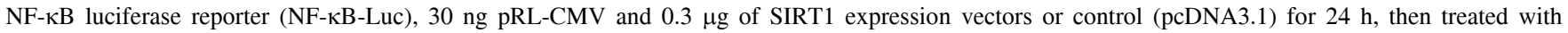
PMA/Io (10 ng/ml PMA plus $0.25 \mu \mathrm{M}$ Io) or vehicle DMSO for $3 \mathrm{~h}$. The relative luciferase activities are presented as the mean \pm SD of triplicate samples and are representative of three independent experiments. **, $P<0.01$.

Table S1 Oligonucleotide sequences used in this study

The supporting information is available online at life.scichina.com and www.springerlink.com. The supporting materials are published as submitted, without typesetting or editing. The responsibility for scientific accuracy and content remains entirely with the authors. 